\title{
Mixed mode stress intensity factors of slanted edge cracked plate with hole subjected to various in-plane loadings using XFEM
}

\author{
Khubilal Khatri and Achchhe Lal* \\ Department of Mechanical Engineering, \\ S.V. National Institute of Technology, \\ Ichchhanath, Surat - 395007, Gujarat, India \\ Email: klkhatri2010@gmail.com \\ Email: lalachchhe@yahoo.co.in \\ *Corresponding Author
}

\begin{abstract}
This manuscript presents the fracture behaviour of a slanted edge cracked plate with a circular hole subjected to various in-plane loadings, such as tensile, shear and combined (tensile and shear) loadings, through the mixed mode stress intensity factors (MMSIF). There is a lack of work in the direction of calculating the fracture behaviour of the slanted crack under the influence of a hole subjected to the various in-plane loadings. In this work, the crack is modelled using extended finite element method (XFEM) under plane strain condition using MATLAB. The MMSIFs are increased with the increment in the crack length but as the crack angle is increased the Mode-I SIFs are decreased whereas Mode-II SIFs are increased. The SIFs are critical for the plate with a hole as compared to the plate without a hole. The values of MMSIFs are the highest for the plate under combined loading and the least under tensile loading.
\end{abstract}

Keywords: extended finite element method; XFEM; mixed mode stress intensity factors; MMSIF; slanted edge crack; plate with a circular hole; in-plane loadings.

Reference to this paper should be made as follows: Khatri, K. and Lal, A. (2019) 'Mixed mode stress intensity factors of slanted edge cracked plate with hole subjected to various in-plane loadings using XFEM', Int. J. Materials and Structural Integrity, Vol. 13, Nos. 1/2/3, pp.110-124.

Biographical notes: Khubilal Khatri has been pursuing his $\mathrm{PhD}$ in Mechanical Engineering from the S.V. National Institute of Technology, Surat, Gujarat, India. His current research area is fracture mechanics. He has been pursuing his $\mathrm{PhD}$ under supervision of Dr. Achchhe Lal.

Achche Lal is an Assistant Professor in Mechanical Engineering at S.V. National Institute of Technology, Surat, Gujarat, India. He has published research papers in various international/national journals and conferences.

This paper is a revised and expanded version of a paper entitled 'SIFs of slanted edge cracked plate with hole subjected to various in-plane loadings' presented at 3rd Indian Conference on Applied Mechanics-2017, Motilal Nehru National Institute of Technology, Allahabad, U.P., India, 5-7 July 2017. 


\section{Introduction}

A big structure cannot be designed without joints and the joints always need holes for riveting or using nuts and bolts (Khatri and Lal, 2018). Under fatigue loading, the fracture behaviour analysis of such structure is necessary for avoiding unexpected failure as the stress concentrations are much critical around the periphery of the holes. The types of loading, shapes, crack length and influence of stress concentration of holes play an important role in the rate of crack propagation as the stress distribution and stresses around the crack tip are increased very rapidly. The intensity of these stresses is responsible to propagate the cracks and hence, it is necessary to evaluate SIF against crack growth under different types of external loadings for predicting and analysing the fracture behaviour of the structure.

Various numerical tools have been used to evaluate the SIF, of which the finite element method (FEM) (Abdelaziz and Hamouine, 2008) is one of the powerful tools and is being used in the various fields of sciences, engineering, and industrial design applications. However, for the modelling of the evolving discontinuities using FEM is cumbersome due to need update meshing in each step of crack growth and always there will be inaccuracy to measure singular behaviour near crack tips. Hence for modelling of such types of problems, the extended finite element method (XFEM) has been used successfully based on the partition of unity by providing extra functions as crack faces and crack tips. In this direction, Belytschko and Black (1999) present a minimal remeshing FEM for crack growth. They use discontinuous enrichment functions with the finite element approximation for showing the presence of the crack. Also, Möes et al. (1999) present an improvement of a new technique for modelling cracks in the finite element framework using a standard displacement-based approximation enriched near a crack. Sukumar et al. (2001) propose a methodology for modelling holes and inclusions without meshing. The level set method (LSM) is coupled with XFEM through the partition of unity method (PUM). Also, Pais (2012) explains the concept and methodology of enrichment in details based on the concept of PUM and LSM with XFEM for modelling cracks, holes, and inclusions using MATLAB. Also, Kirthan et al. (2016) have evaluated SIFs Mode-I crack propagation for different crack lengths using a compact specimen using displacement extrapolation method. Using FEM, Fajdiga and Zafošnik (2015) apply the maximum energy release rate criterion, of the minimum strain energy density (SED) criterion and of the maximum tangential stress (MTS) criterion for evaluating the kink angles for the propagation of the crack in different loading modes. Similarly, Kumar et al. (2015) presents virtual node extended finite element method (VNXFEM) for modelling and simulation of kinked cracks in a single element and after kink in the crack the crack tip simulates under mixed mode loading. Liu et al. (2004) improve XFEM for directly evaluating mixed mode stress intensity factors (SIFs). Also, Pathak et al. (2012) apply element free Galerkin method (EFGM) and XFEM under mode-I and mixed mode loading Conditions for simulating bi-material interfacial cracks. The modified interaction integral approach is used for evaluating the mixed mode SIFs for bi-material interface cracks. Similarly, Bhattacharya et al. (2013) investigate fatigue crack growth of interfacial cracks in bi-layered materials under thermos mechanical loadings by evaluating SIFs using XFEM. The cyclic, thermal and mixed mode loadings are applied for predicting the fatigue life of the interface crack using the Paris law of fatigue crack growth. 
The fracture behaviour of slanted edge cracked plate under tensile and bending loadings is analysed by evaluating mixed mode stress intensity factors (MMSIFs) using ANSYS with FEM by Ismail et al. (2017) and Fayed (2017). The literature review shows that very little work is performed for calculating the MMSIFs of a slanted edge crack plate with a hole subjected to various in-plane loadings using XFEM. In this work, The MMSIFs of a slanted edge cracked plate with a hole under the influence of different crack angles and lengths subjected to tensile, shear and combined (tensile and shear) loadings are calculated using XFEM using MATLAB through numerical examples.

\section{XFEM formulation}

For the modelling of the cracks and voids, the XFEM displacement vector is represented by Möes et al. (1999) and Sukumar et al. (2001) as:

$$
\begin{aligned}
u^{h}(x)= & \sum_{i=1}^{n} N_{i}(x)[\bar{u}_{i}+\underbrace{\left[H 1(x)-H 1\left(x_{i}\right)\right] a_{i}}_{\text {for crack face }} \\
& +\underbrace{\sum_{\alpha=1}^{n}\left[\phi_{\alpha}(x)-\phi_{\alpha}\left(x_{i}\right)\right] b_{i}^{\alpha}}_{\text {for crack up enrichment }}+\underbrace{\left[H 2(x)-H 2\left(x_{i}\right)\right] c_{i}}_{\text {for hole }}]
\end{aligned}
$$

where $n$ is the sets of all nodes in the mesh, $N_{i}(x)$ are conventional finite element nodal shape functions, $\bar{u}_{i}$ are the displacements of the nodes associated with the continuous part of the solution and $H 1(x)$ is the discontinuous or Heaviside function which deals with the discontinuous jump across crack faces. The Heaviside jump function $H 1(x)$ is used for the element which is completely cut by a crack, and it takes a constant value and is equal to +1 on one side and -1 on another side of the crack. The $a_{i}$ and $b_{i}^{\alpha}$ are nodal enriched degree of freedom vectors associated with $H 1(x)$ and crack tip enrichment, respectively. $H 2(x)$ is a discontinuous function for holes and its value is 0 for the inside of the hole and 1 for the rest of the part and the $c_{i}$ is the degree of freedom associated with $H 2(x) . \phi_{\alpha}(x)$ is a near-tip asymptotic function representing crack tip fields. It is denoted by:

$$
\phi_{\alpha}(x)=\sqrt{r}\left[\sin \left(\frac{\theta}{2}\right), \cos \left(\frac{\theta}{2}\right), \sin (\theta) \cos \left(\frac{\theta}{2}\right), \sin (\theta) \sin \left(\frac{\theta}{2}\right)\right]
$$

where $(r, \theta)$ are polar coordinates in the local crack tip coordinate system.

\subsection{LSM for cracks}

The LSM is used as a tool for tracking the interfaces. In LSM, the interface surface is represented by zero level set function and is defined by a scalar function within the domain. The displacement discontinuities in the interior part of the crack are modelled by an extrinsic function based on Heaviside function $H 1(x)$. It is defined as:

$$
H 1(x)=\operatorname{sign}(\phi(x))= \begin{cases}1 & \text { if } \phi(x) \geq 0 \\ -1 & \text { otherwise }\end{cases}
$$


where the sign ( $)$ is signed distance function. The Heaviside function $H 1(x)$ has the value +1 above the crack and $\square 1$ below the crack.

\subsection{LSM for circular holes}

Voids or holes require the static interface, so the representation of circular holes by the level set function using the Heaviside function as a discontinuous function is:

$$
H 2(x)=\operatorname{sign}(\chi(x))= \begin{cases}1 & \text { if } \chi(x) \geq 0 \\ 0 & \text { otherwise }\end{cases}
$$

\subsection{Evaluation of SIF}

For evaluating stress intensity factor, the auxiliary displacement, stress, strain field must be selected as the crack tip asymptotic field. The relationship between SIF and $J$-integral for genera mixed-mode problems in two dimensions are presented by Möes et al. (1999) and can be written as:

$$
J=\frac{K_{I}^{2}}{E^{*}}+\frac{K_{I I}^{2}}{E^{*}}, \text { where } E^{*}= \begin{cases}E & \text { for plane stress } \\ \frac{E}{1-v^{2}} & \text { for plane strain }\end{cases}
$$

Now, the $J$-integral:

$$
J=\int_{\Gamma}\left(W n_{1}-\sigma_{j k} n_{j} \frac{\partial u_{k}}{\partial x_{1}}\right) d \Gamma
$$

where $W$ is the SED. Equation (6) can be written in the equivalent form using Dirac delta which is easier to implement in finite element code as:

$$
J=\int_{\Gamma}\left(W \delta_{1 j}-\sigma_{j i} \frac{\partial u_{i}}{\partial x_{1}}\right) n_{j} d \Gamma
$$

where $\Gamma$ is the area inside the contour, $\sigma_{i j}$ is stress tensor and $u_{i}$ is displacement field vector.

Here, two sates of cracked body are considered. State $1,\left(\sigma_{i j}^{(1)}, \varepsilon_{i j}^{(1)}, u_{i j}^{(1)}\right)$, corresponds to the present state and state $2,\left(\sigma_{i j}^{(2)}, \varepsilon_{i j}^{(2)}, u_{i j}^{(2)}\right)$, is an auxiliary state. The J-integral for the sum of the two states is:

$$
J^{(1+2)}=\int_{\Gamma}\left[\frac{1}{2}\left(\sigma_{i j}^{(1)}+\sigma_{i j}^{(2)}\right)\left(\varepsilon_{i j}^{(1)}+\varepsilon_{i j}^{(2)}\right) \delta_{1 j}-\left(\sigma_{i j}^{(1)}+\sigma_{i j}^{(2)}\right) \frac{\partial\left(u_{i}^{(1)}+u_{i}^{(2)}\right)}{\partial x_{1}}\right] n_{j} d \Gamma
$$

Now, the equation (8) is separated in XFEM state $J^{(1)}$, the auxiliary state $J^{(2)}$ and interaction state $M^{(1,2)}$ and can be written as:

$$
J^{(1+2)}=J^{(1)}+J^{(2)}+M^{(1,2)}
$$




$$
M^{(1,2)}=\int_{\Gamma}\left(W^{(1,2)} \delta_{1, j}-\sigma_{i j}^{(1)} \frac{\partial u_{i}^{(2)}}{\partial x_{1}}-\sigma_{i j}^{(2)} \frac{\partial u_{i}^{(1)}}{\partial x_{1}}\right) n_{j} d \Gamma
$$

where $W^{(1,2)}$ is interaction SED and is denoted as:

$$
W^{(1,2)}=\sigma_{i j}^{(1)} \varepsilon_{i j}^{(2)}=\sigma_{i j}^{(2)} \varepsilon_{i j}^{(1)}
$$

Now by equation (9), we have:

$$
J^{(1+2)}=J^{(1)}+J^{(2)}+\frac{2}{E^{*}}\left(K_{I}^{(1)} K_{I}^{(2)}+K_{I I}^{(1)} K_{I I}^{(2)}\right)
$$

Again, by comparing the equations (9) and (12), we have:

$$
M^{(1,2)}=\frac{2}{E^{*}}\left(K_{I}^{(1)} K_{I}^{(2)}+K_{I I}^{(1)} K_{I I}^{(2)}\right)
$$

The SIFs for the XFEM state $K_{I}^{(1)}$ and $K_{I I}^{(1)}$ are evaluated by putting $K_{I}^{(2)}=1$ and $K_{I I}^{(2)}=0$ in the equation (13), we have:

$$
K_{I}^{(1)}=\frac{M^{(1, \text { Model I })} E^{*}}{2}
$$

Similarly, by putting $K_{I}^{(2)}=1$ and $K_{I I}^{(2)}=0$ in equation (13), we have:

$$
K_{I I}^{(1)}=\frac{M^{(1, \text { Model II) }} E^{*}}{2}
$$

where $M^{(1, \text { Model I) }}$ and $M^{(1, \text { Model II) }}$ are interaction integrals.

\subsection{Evaluation of kink angle of the crack}

The crack propagation criterion is specified and explained by Möes et al. (1999). For the evaluation of the kink angle of the crack, the maximum circumferential stress criterion is used. The criterion explains that the crack kinks in such direction so that the asymptotic near tip circumferential stress is maximum. For the mixed mode loading condition, the asymptotic near tip circumferential and shear stresses are:

$$
\begin{aligned}
\left\{\begin{array}{l}
\sigma_{\theta \theta} \\
\tau_{r \theta}
\end{array}\right\}= & \frac{K_{I}}{\sqrt{2 \pi r}} \frac{1}{4}\left\{\begin{array}{l}
3 \cos (\theta / 2)+\cos (3 \theta / 2) \\
\sin (\theta / 2)+\sin (3 \theta / 2)
\end{array}\right\} \\
& +\frac{K_{I I}}{\sqrt{2 \pi r}} \frac{1}{4}\left\{\begin{array}{l}
-3 \sin (\theta / 2)-3 \sin (3 \theta / 2) \\
\cos (\theta / 2)+3 \cos (3 \theta / 2)
\end{array}\right\}
\end{aligned}
$$

So, the kink angle can be predicted by putting shear stress to zero. The further solution of the equation provides:

$$
K_{I} \sin \left(\theta_{c}\right)+K_{I I}\left(3 \cos \left(\theta_{c}\right)-1\right)=0
$$

therefore, the king angle for the crack propagation is: 


$$
\theta_{k}=2 \arctan \frac{1}{4}\left(K_{I} / K_{I I} \pm \sqrt{\left(K_{I} / K_{I I}\right)^{2}+8}\right)
$$

\section{Results and discussion}

In this paper, first, the validation of the MATLAB coding is performed by evaluating the SIFs, $K_{I}$, of a rectangular edge cracked plate of the dimensions $7 \mathrm{~mm} \times 16 \mathrm{~mm}$ with different crack lengths in mm, $a=1,1.5,2.0$ and 3.5 using a mesh size of $140 \times 320$ under a tensile stress of $\sigma=1.0 \mathrm{~Pa}$. The results are compared with the results evaluated using analytical equation available in the literature (Chen et al., 2012) as shown in Table 1. The exact solution of $K_{I}$ is represented by:

$$
K_{I}=F \sigma \sqrt{\pi a}
$$

here, $F$ is a geometrical correction factor and it is denoted as:

$$
F=1.12-0.231(a / W)+10.55(a / W)^{2}-21.72(a / W)^{3}+30.39(a / W)^{4}
$$

Similarly, the MMSIFs, $K_{I}$ and $K_{I I}$, of a rectangular edge cracked plate with the same geometric configuration, material properties, boundary conditions under a shear stress of $\tau=1.0 \mathrm{~Pa}$ with a crack length of $a=3.5 \mathrm{~mm}$. The results are in good agreement with

\begin{tabular}{|c|c|c|c|c|c|c|}
\hline \multirow{2}{*}{ Mesh size } & \multicolumn{6}{|c|}{ SIFs, $K_{I},(P a \sqrt{m m})$} \\
\hline & \multicolumn{3}{|c|}{ Analytical result } & \multicolumn{3}{|c|}{ Present results } \\
\hline \multirow[t]{2}{*}{$140 \times 320$} & $a=1$ & $a=1.5$ & $a=2$ & $a=1$ & $a=1.5$ & $a=2$ \\
\hline & 2.219 & 3.051 & 4.039 & 2.219 & 3.040 & 4.032 \\
\hline
\end{tabular}
results available in the literature (Chen et al., 2012) as shown in Table 2.

Table 1 Validation of an edge cracked plate under tensile loading

\begin{tabular}{|c|c|c|c|c|}
\hline \multirow{3}{*}{ Mesh size } & \multicolumn{4}{|c|}{ SIFs } \\
\hline & \multicolumn{2}{|c|}{ Chen et al. (2012) } & \multicolumn{2}{|c|}{ Present results } \\
\hline & $K_{I},(P a \sqrt{m m})$ & $K_{I I},(P a \sqrt{m m})$ & $K_{I},(P a \sqrt{m m})$ & $K_{I I},(P a \sqrt{m m})$ \\
\hline $140 \times 320$ & 34.0000 & 4.5500 & 34.0144 & 4.6500 \\
\hline
\end{tabular}

Table 2 Validation of an edge cracked plate under shear loading

Here, for the edge cracked plate under tensile or shear loading, the bottom edge of the plate has rollers and the bottom left corner is fixed. The tensile and shear loadings are applied on the top edge of the plate in $y$ - and $x$-direction, respectively.

\subsection{A slanted edge cracked plate with a circular hole subject to various in-plane loadings}

Now, the numerical examples of an isotropic finite rectangular slanted edge cracked plate with a circular hole subjected to various in-plane loadings is solved and the evaluated results in terms of MMSIFs, $K_{I}$ and $K_{I I}$, are compared with those of a finite slanted edge 
cracked plate without any hole with same dimension, material properties, loading conditions and crack configurations.

The dimensions of the plate are the same as mentioned earlier with the different crack lengths of $a=1.5,2.0$ and $2.5 \mathrm{~mm}$, crack angles of $\alpha=0^{\circ}, 15^{\circ}, 30^{\circ}$ and $45^{\circ}$, radius of hole, $R=0.5 \mathrm{~mm}$ with mechanical properties such as Young's modulus, $E=3 \times 10^{7} \mathrm{~Pa}$, Poisson's ratio $v=0.3$, subjected to various in-plane loading such as tensile $(\sigma=1.0 \mathrm{~Pa})$, shear $(\tau=1.0 \mathrm{~Pa})$, and combined $(\sigma=\tau=1.0 \mathrm{~Pa})$. For the tensile, shear and combined loading, the displacements along the $y$-axis are fixed at the bottom edge, and the plate is clamped at the bottom left corner, and then the loading is applied on the top edge of the plate.

\subsubsection{A slanted edge cracked plate with a circular hole subject to tensile loading}

Figure 1 shows a line diagram and FE meshing of an isotropic finite slanted edge cracked plate with a circular hole subjected to tensile loadings. The evaluated values of the MMSIFs, $K_{I}$ and $K_{I I}$, of the slanted edge cracked plate with hole under tensile loading for different crack length $(a)$ and angles $(\alpha)$ using XFEM are shown in Table 3.

Figure 1 (a) A line diagram (b) An FE meshing of an isotropic finite slanted edge cracked plate with a circular hole under in-plane tensile loading (see online version for colours)

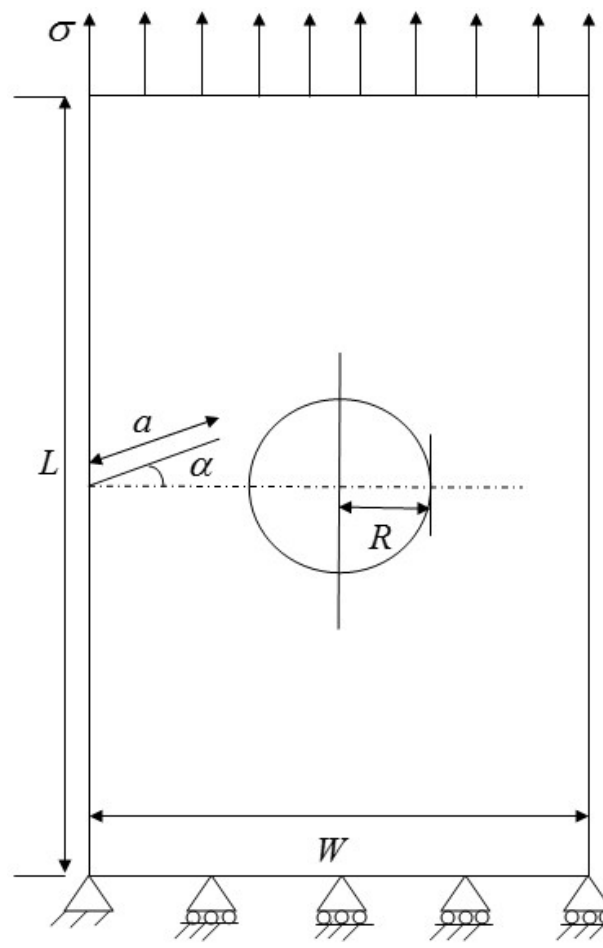

(a)

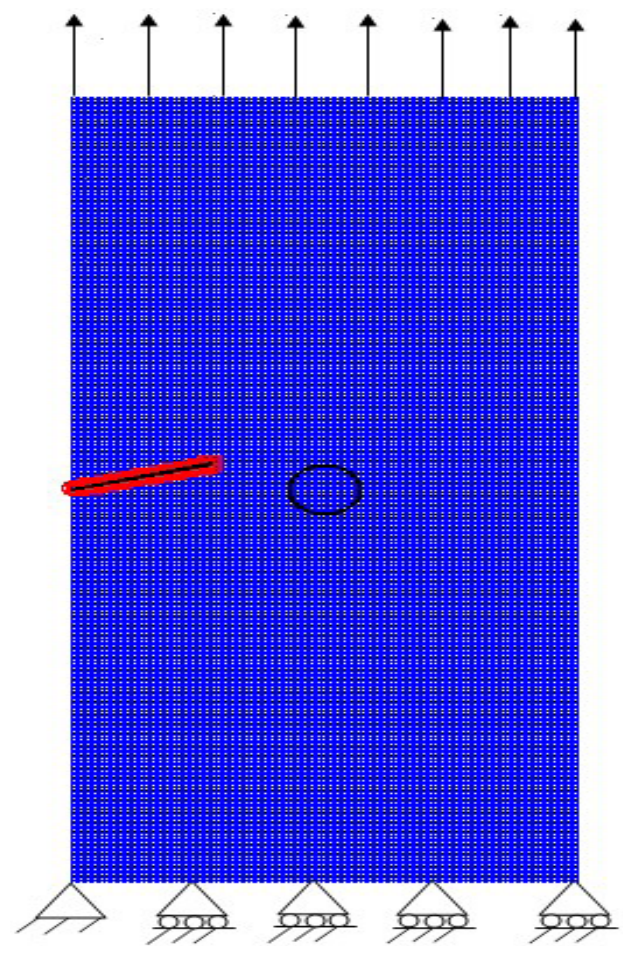

(b)

Figure 2 shows the fracture behaviour of a slanted edge cracked plate with a circular hole and without any hole under tensile loading in terms of MMSIFs, $K_{I}$ and $K_{I I}$, with different crack lengths and crack angles. 
Table 3 MMSIFs of a slanted edge cracked plate with a circular hole under tensile loading

\begin{tabular}{|c|c|c|c|c|c|c|c|c|c|}
\hline \multirow[b]{2}{*}{$\alpha$} & \multirow{2}{*}{$a$} & $K_{I}$ & $K_{I I}$ & & $K_{I}$ & $K_{I I}$ & \multirow[b]{2}{*}{$a$} & $K_{I}$ & $K_{I I}$ \\
\hline & & \multicolumn{2}{|c|}{$(P a \sqrt{m m})$} & & \multicolumn{2}{|c|}{$(P a \sqrt{m m})$} & & \multicolumn{2}{|c|}{$(P a \sqrt{m m})$} \\
\hline $0^{\circ}$ & $1.0 \mathrm{~mm}$ & 2.265 & 0.001 & $1.5 \mathrm{~mm}$ & 3.174 & 0.001 & $2.0 \mathrm{~mm}$ & 4.387 & 0.001 \\
\hline $15^{\circ}$ & & 2.147 & 0.339 & & 3.00 & 70.449 & & 4.130 & 0.589 \\
\hline $30^{\circ}$ & & 1.830 & 0.596 & & 2.527 & 0.798 & & 3.348 & 1.060 \\
\hline $45^{\circ}$ & & 1.373 & 0.706 & & 1.843 & 0.936 & & 2.329 & 1.196 \\
\hline $60^{\circ}$ & & 0.877 & 0.645 & & 1.143 & 0.832 & & 1.389 & 1.020 \\
\hline $75^{\circ}$ & & 0.422 & 0.424 & & 0.542 & 0.535 & & 0.641 & 0.634 \\
\hline $85^{\circ}$ & & 0.029 & 0.271 & & 0.093 & 0.303 & & 0.246 & 0.303 \\
\hline
\end{tabular}

From Figure 2, as the crack length is increased the values of MMSIFs, $K_{I}$ and $K_{I I}$, of the slanted edge cracked plate with and without a hole are also increased. But for particular crack length, as the crack angle is increased, the values of Mode-I SIFs, $K_{I}$, are continuously decreased from crack angles $\alpha=0^{\circ}$ to $85^{\circ}$ whereas the values of Mode-II SIFs, $K_{I I}$, are increased continuously from crack angles $\alpha=0^{\circ}$ to $45^{\circ}$ and then are decreased from crack angles $\alpha=45^{\circ}$ to $85^{\circ}$ for all crack lengths.

The values of Mode-I SIFs, $K_{I}$, are the highest and the lowest at crack angles $\alpha=0^{\circ}$ and $85^{\circ}$, respectively whereas the values of Mode-II SIFs, $K_{I I}$, are the lowest and the highest at crack angles $\alpha=0^{\circ}$ and $45^{\circ}$, respectively. Further, as the crack angle is increased, the gaps between the curves of the values of Mode-I SIF, $K_{I}$, are continuously decreased whereas the gaps between the curves of the values of Mode-II SIFs, $K_{I I}$, are continuously increased from crack angles $\alpha=0^{\circ}$ to $45^{\circ}$ and then are decreased from crack angles $\alpha=45^{\circ}$ to $85^{\circ}$ for all the crack lengths.

Figure 2 Behaviour of MMSIFs, (a) $K_{I}$ and (b) $K_{I I}$, of a slanted edge cracked plates with a circular hole and without hole subjected to tensile loading (see online version for colours)

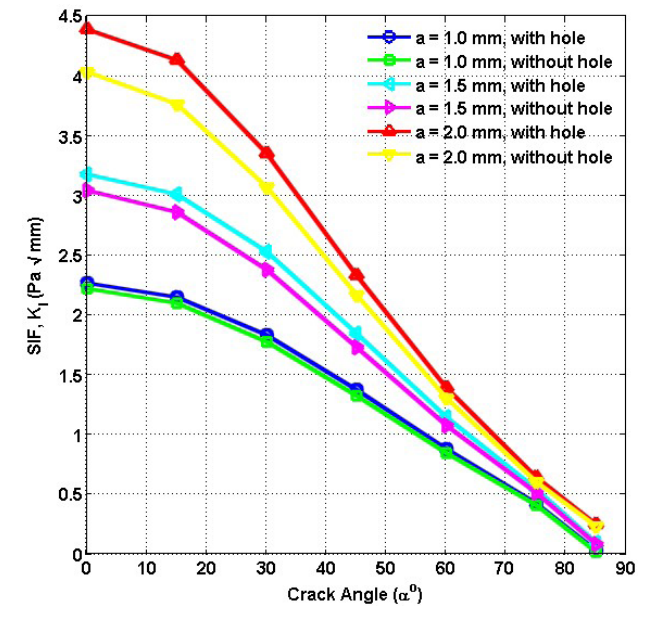

(a)

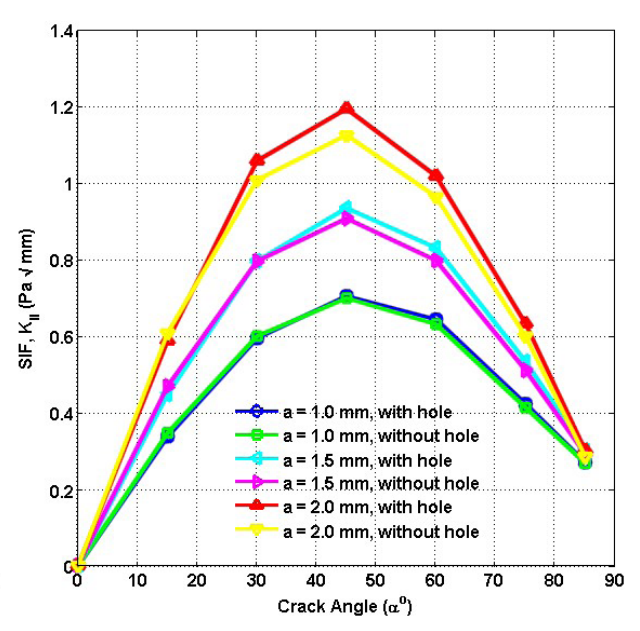

(b) 


\subsubsection{A slanted edge cracked plate with a circular hole subject to shear loading}

Figure 3 shows a line diagram and FE meshing of an isotropic finite slanted edge cracked plate with a circular hole subjected to shear loading. The evaluated values of the MMSIFs, $K_{I}$ and $K_{I I}$, of the slanted edge cracked plate with hole under shear loading for different crack length $(a)$ and angles $(\alpha)$ using XFEM are shown in Table 4.

Figure 3 (a) A line diagram (b) An FE meshing of an isotropic finite slanted edge cracked plate with a circular hole under in-plane shear loading (see online version for colours)

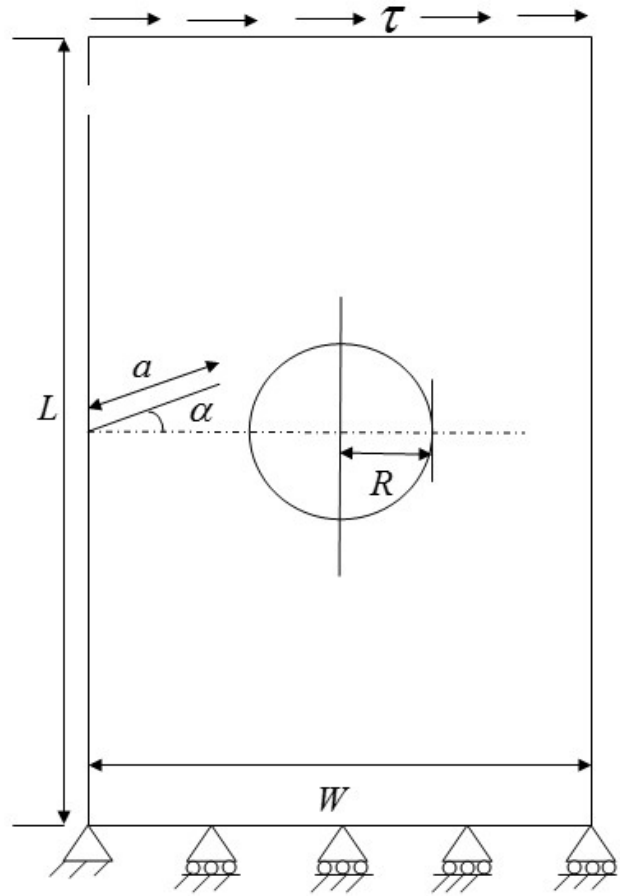

(a)

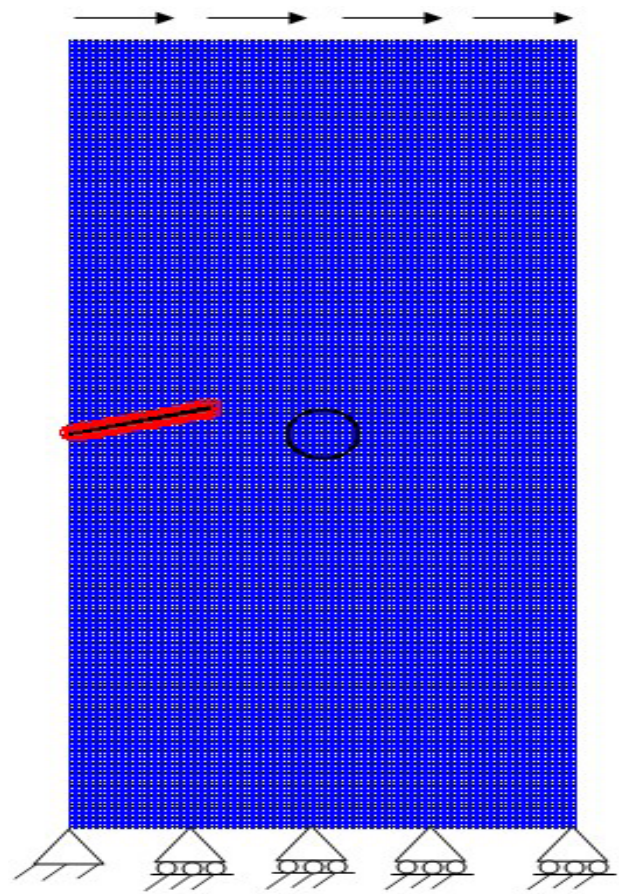

(b)

Figure 4 shows the fracture behaviour of a slanted edge cracked plate with a circular hole and without any hole under shear loading in terms of MMSIFs, $K_{I}$ and $K_{I I}$, with different crack lengths and crack angles.

From Figure 4, for a particular crack length, as the crack length is increased the values of Mode-I SIFs, $K_{I}$, of the slanted edge cracked plate with hole are increased continuously from crack angles $\alpha=0^{\circ}$ to $75^{\circ}$ for crack length $a=1.0 \mathrm{~mm}$ and $1.5 \mathrm{~mm}$ but are decreased from crack angles $\alpha=0^{\circ}$ to $90^{\circ}$ for crack length $a=2.0 \mathrm{~mm}$. The values of Mode-II SIFs, $K_{I I}$, of the plate with and without hole are increased continuously from crack angles $\alpha=0^{\circ}$ to $45^{\circ}$ and are decreased from crack angles $\alpha=45^{\circ}$ to $75^{\circ}$ for crack length $a=1.0 \mathrm{~mm}$. The SIFs are further increased from crack angles $\alpha=75^{\circ}$ to $85^{\circ}$. 
Table 4 MMSIFs of a slanted edge cracked plate with a circular hole under shear loading

\begin{tabular}{|c|c|c|c|c|c|c|c|c|c|}
\hline \multirow[b]{2}{*}{$\alpha$} & \multirow[b]{2}{*}{$a$} & $K_{I}$ & $K_{I I}$ & \multirow[b]{2}{*}{$a$} & $K_{I}$ & $K_{I I}$ & \multirow[b]{2}{*}{$u$} & $K_{I}$ & $K_{I I}$ \\
\hline & & \multicolumn{2}{|c|}{$(P a \sqrt{m m})$} & & \multicolumn{2}{|c|}{$(P a \sqrt{m m})$} & & \multicolumn{2}{|c|}{$(P a \sqrt{m m})$} \\
\hline $0^{\circ}$ & $1.0 \mathrm{~mm}$ & 12.780 & 1.208 & $1.5 \mathrm{~mm}$ & 16.142 & 2.085 & $2.0 \mathrm{~mm}$ & 20.137 & 3.133 \\
\hline $15^{\circ}$ & & 11.267 & 2.882 & & 13.713 & 3.939 & & 16.406 & 5.034 \\
\hline $30^{\circ}$ & & 9.071 & 3.808 & & 10.639 & 4.726 & & 12.226 & 5.571 \\
\hline $45^{\circ}$ & & 6.619 & 3.948 & & 7.505 & 4.624 & & 8.238 & 5.201 \\
\hline $60^{\circ}$ & & 4.260 & 3.400 & & 4.724 & 3.823 & & 4.997 & 4.126 \\
\hline $75^{\circ}$ & & 2.152 & 2.235 & & 2.398 & 2.472 & & 2.505 & 2.600 \\
\hline $85^{\circ}$ & & 3.617 & 2.538 & & 1.934 & 2.319 & & 1.063 & 1.323 \\
\hline
\end{tabular}

Figure 4 Behaviour of MMSIFs, (a) $K_{I}$ and (b) $K_{I I}$, of a slanted edge cracked plates with a circular hole and without hole subjected to shear loading (see online version for colours)

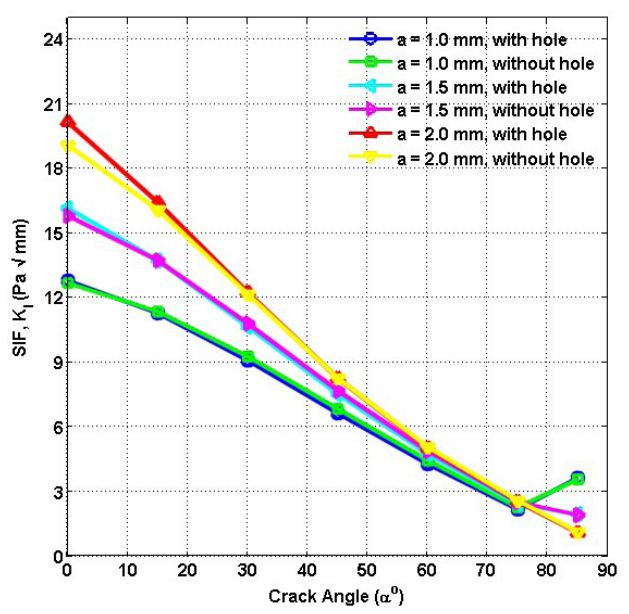

(a)

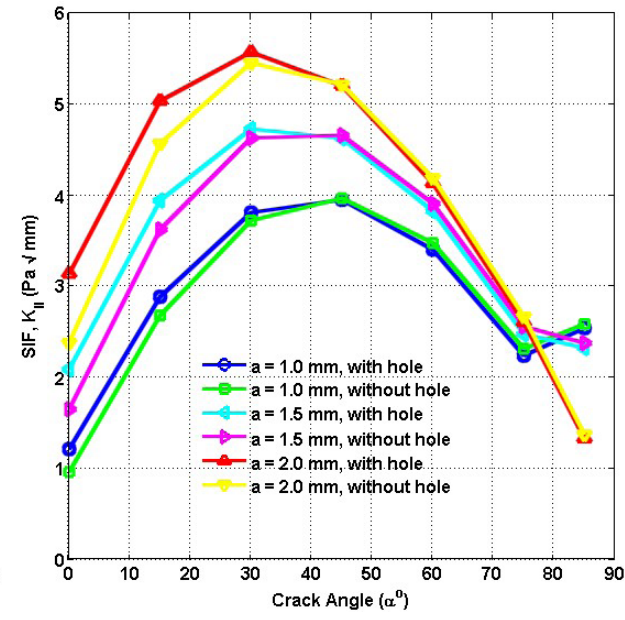

(b)

For crack lengths $a=1.5$ and 2.0, the values of Mode-II SIFs, $K_{I I}$, of the plates are increased continuously from crack angles $\alpha=0^{\circ}$ to $30^{\circ}$ and are decreased from crack angles $\alpha=30^{\circ}$ to $75^{\circ}$. The SIFs are increased and decreased from crack angles $\alpha=75^{\circ}$ to $85^{\circ}$ for crack lengths $a=1.5$ and 2.0 , respectively.

The values of Mode-I SIFs, $K_{I}$, are the highest and the lowest at crack angles $\alpha=0^{\circ}$ and $90^{\circ}$ for crack lengths $a=1.5$ and 2.0, respectively but are the highest and the lowest at crack angles $\alpha=0^{\circ}$ and $75^{\circ}$ for crack lengths $a=1.0$. The values of Mode-II SIFs, $K_{I I}$, are the lowest and the highest at crack angles $\alpha=0^{\circ}$ and $45^{\circ}$, respectively for crack length $a=1 \mathrm{~mm}$. But, these values are the lowest and the highest at crack angles $\alpha=0^{\circ}$ and $30^{\circ}$, respectively for crack lengths $a=1.5$ and $2.0 \mathrm{~mm}$. Further, as the crack angle is 
increased the gaps between the curves of the values of MMSIFs, $K_{I}$ and $K_{I I}$, of the respective plates are continuously decreased from crack angles $\alpha=0^{\circ}$ and $75^{\circ}$. The values of MMSIFs of the slated edge cracked plate with and without hole under shear loading are much critical as compared to the same plates under tensile loadings, respectively.

\subsubsection{A slanted edge cracked plate with a circular hole subject to combined loading}

Figure 5 shows a line diagram and FE meshing of an isotropic finite slanted edge cracked plate with a circular hole subjected to combined loading. The evaluated values of the MMSIFs, $K_{I}$ and $K_{I I}$, of the slanted edge cracked plate with hole under combined loading for different crack length $(a)$ and angles $(\alpha)$ using XFEM are shown in Table 5.

Figure 5 (a) A line diagram (b) An FE meshing of a finite slanted edge cracked plate with a circular hole under combined loading (see online version for colours)

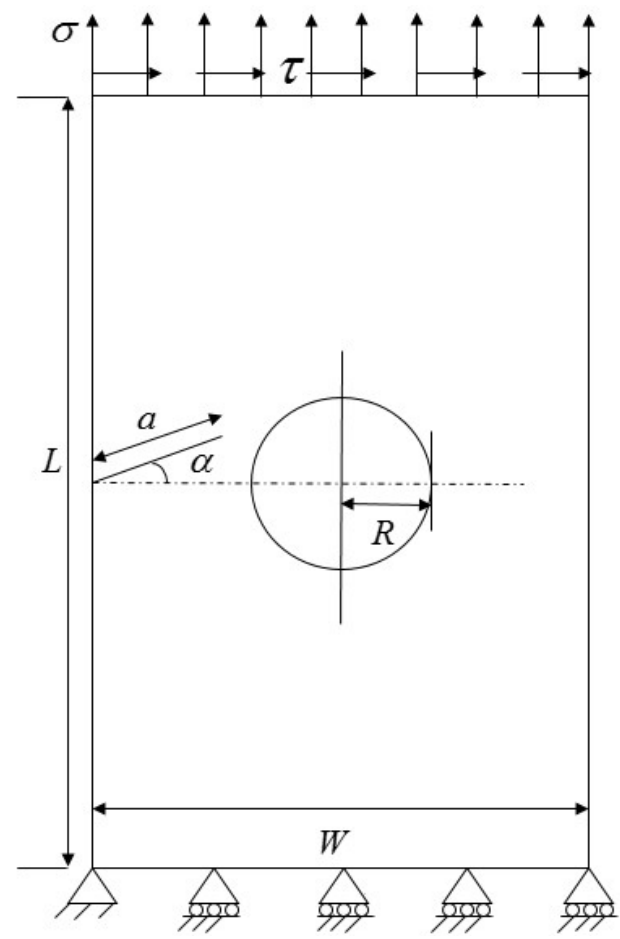

(a)

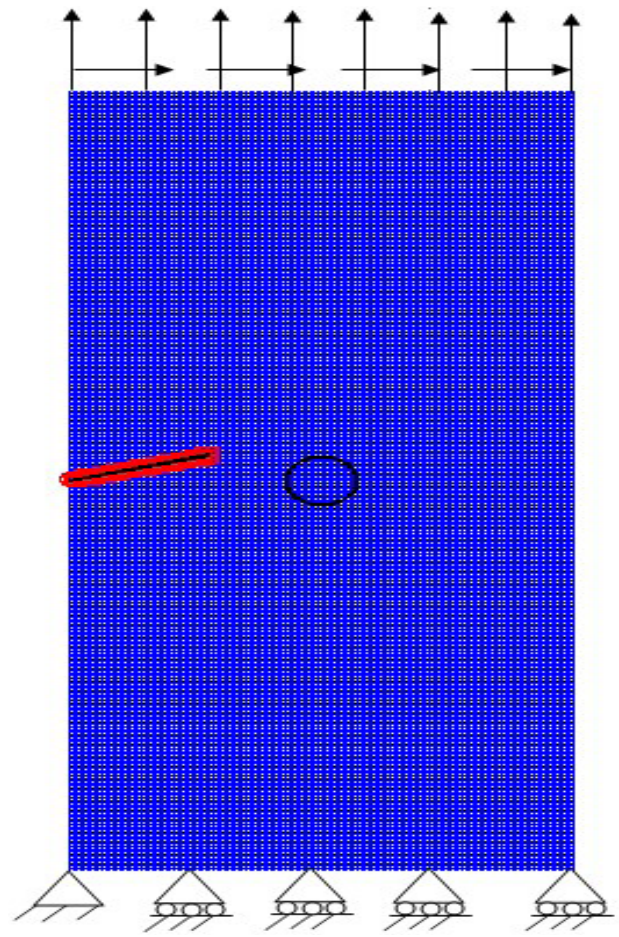

(b)

Figure 6 shows the fracture behaviour of a slanted edge cracked plate with a circular hole and without any hole under combined loading in terms of MMSIFs, $K_{I}$ and $K_{I I}$, with different crack lengths and crack angles.

From Figure 6, the fracture behaviour of the plates under combined loading is similar but much critical as compared to that of the plates under shear loading. 
Table 5 MMSIFs of a slanted edge cracked plate with a circular hole under combined loading

\begin{tabular}{|c|c|c|c|c|c|c|c|c|c|}
\hline \multirow[b]{2}{*}{$\alpha$} & \multirow[b]{2}{*}{$a$} & $K_{I}$ & $K_{I I}$ & \multirow[b]{2}{*}{$a$} & $K_{I}$ & $K_{I I}$ & \multirow{2}{*}{$a$} & $K_{I}$ & $K_{I I}$ \\
\hline & & \multicolumn{2}{|c|}{$(P a \sqrt{m m})$} & & \multicolumn{2}{|c|}{$(P a \sqrt{m m})$} & & \multicolumn{2}{|c|}{$(P a \sqrt{m m})$} \\
\hline $0^{\circ}$ & $1.0 \mathrm{~mm}$ & 15.045 & 1.209 & $1.5 \mathrm{~mm}$ & 19.317 & 2.086 & $2.0 \mathrm{~mm}$ & 24.524 & 3.135 \\
\hline $15^{\circ}$ & & 13.414 & 3.221 & & 16.721 & 4.388 & & 20.536 & 5.624 \\
\hline $30^{\circ}$ & & 10.901 & 4.404 & & 13.166 & 5.524 & & 15.574 & 6.631 \\
\hline $45^{\circ}$ & & 7.992 & 4.654 & & 9.347 & 5.560 & & 10.567 & 6.397 \\
\hline $60^{\circ}$ & & 5.137 & 4.043 & & 5.867 & 4.655 & & 6.386 & 5.149 \\
\hline $75^{\circ}$ & & 2.573 & 2.659 & & 2.939 & 3.008 & & 3.145 & 3.234 \\
\hline $85^{\circ}$ & & 3.589 & 2.809 & & 1.843 & 2.623 & & 1.308 & 1.626 \\
\hline
\end{tabular}

\subsection{Comparison between the behaviour of MMSIFs of a slanted edge cracked plate with and without a circular hole subject to various in-plane loadings}

Under all loading conditions, the behaviour of the values of MMSIFs, $K_{I}$ and $K_{I I}$, of the slanted edge cracked plate with and without hole are similar as mentioned in Subsection 3.1

Figure 6 Behaviour of MMSIFs, (a) $K_{I}$ and (b) $K_{I I}$, of a slanted edge cracked plates with a circular hole and without hole subjected to combined loading (see online version for colours)

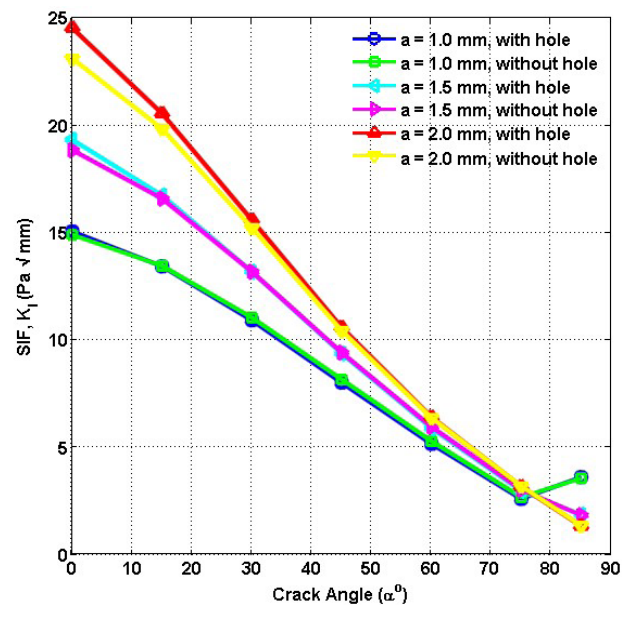

(a)

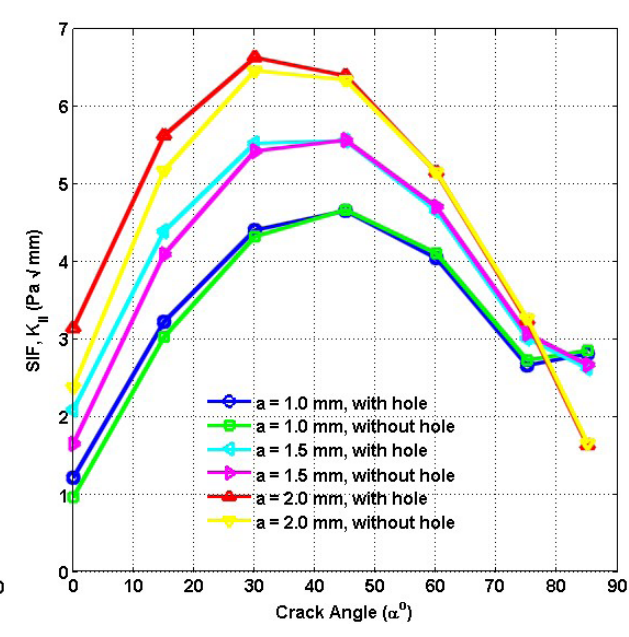

(b)

Under tensile loading (Figure 2), as the crack length is increased the gaps between the Mode-I SIFs, $K_{I}$, of both the plates are increased and values are higher for the plate with a hole because the influence of the stress concentration of the circular hole on the crack tip is higher for the longer crack length as the tip of the longer crack is much near to the stress concentration zone of the circular hole. As the crack angle is increased the gap between the values of Mode-I SIF, $K_{I}$, of both the plates are decreased because as the 
crack angle is increased the tip of the crack is moved away from the hole so there is the less influence of the stress concentration of the hole on the crack tip.

Under tensile loading, at crack angle $\alpha=0^{\circ}$, the values of Mode-II SIFs, $K_{I I}$, of both the plates are same (nearly zero) but as the crack angle increased the Mode-II SIFs, $K_{I I}$, of the plate with a hole are increased but nearly same with the plate without hole as for the crack lengths $a=1.0$ and $1.5 \mathrm{~mm}$ from crack angles $\alpha=0^{\circ}$ to $30^{\circ}$.

The Mode-II SIFs, $K_{I I}$, of the plate with hole are higher as compared to those of plate without hole for crack angle $\alpha=45^{\circ}$. Therefore, the influence of the stress concentration of the hole is higher on the tip of the longer crack, i.e., $a=2.0 \mathrm{~mm}$ with higher crack angle $\alpha=45^{\circ}$. Now, The Mode-II SIFs, $K_{I I}$, of both the plates are continuously decreased from crack angles $\alpha=45^{\circ}$ to $85^{\circ}$ and the gaps between the curve of the plate with hole and without hole are also decreased.

Under shear loading (Figure 4), as the crack angle is increased the gap between the values of Mode-I SIF, $K_{I}$, of both the plates are decreased and nearly, there is no (less) gap from the crack angles $\alpha=15^{\circ}$ to $85^{\circ}$. Under shear loading, the influence of the stress concentration of the hole on the crack tip nearly vanishes after crack angle $\alpha=15^{\circ}$.

Under shear loading, at crack angle $\alpha=0^{\circ}$, the gaps between values of Mode-II SIFs, $K_{I I}$, of both the plates are higher but as the crack angle is increased the gaps between these values are decreased to zero (nearly) from crack angle $\alpha=0^{\circ}$ to $45^{\circ}$ and the values of the SIFs are same for both the plates from crack angle $\alpha=45^{\circ}$ to $85^{\circ}$. The values of Mode-II SIFs, $K_{I I}$, of the plate with hole are higher for longer crack and higher crack angle but the influence of the stress concentration on the crack tip is higher on the longer crack having smaller angles.

Under combined loading (Figure 6), the behaviour of the values of MMSIFs, $K_{I}$ and $K_{I I}$, of the slanted edge cracked plate with and without hole are similar as mentioned for the plates under shear loading. The major difference is that the MMSIFs of the plates under the combined loading is much critical as compared to those of the plates under tensile and shear loadings individually.

\subsection{Comparison between the effects of applied various in-plane loadings on the behaviour of MMSIFs of a slanted edge cracked plate with a circular hole}

The values of MMSIFs, $K_{I}$ and $K_{I I}$, of the slanted edge cracked plate with and without hole under combined loadings are much critical as compared to those of the plates under shear and tensile loadings, individually. The slanted edge cracked plate with a hole under tensile loading is geometrically under mixed mode loading condition whereas the same plate under shear loading is geometrically as well as by loading is under mixed mode loading condition. Therefore the effect is multiplied by the influence of the stress concentration of the hole. Under combined loadings, the combined effect of the tensile and shear loading on the edge cracked plate with a hole is higher and the values of MMSIFs, $K_{I}$ and $K_{I I}$, are the most critical as compared to other loading conditions. 


\section{Conclusions}

The MMSIFs, $K_{I}$ and $K_{I I}$, of a slanted edge cracked plate with and without hole are increased with the increment in the crack length but as the crack angle is increased the Mode-I SIFs are decreased for particular crack length whereas Mode-II SIFs are increased. Due to the slanted crack, the fractured plate is the result of the combination of the Mode-I and -II of SIFs. The values of MMSIFs are the highest for the plate under combined loading and the least under tensile loading. The combined loading is critical for particular crack length and crack angle as compared to tensile and shear loadings. Also, the MMSIFs are critical for the plate with hole as compared to the plate without hole because the crack tip is under the influence of stress concentration of the circular hole and the stress intensity on the crack tip is increased. The crack tip of longer crack with smaller angle is highly influenced by stress concentration of the hole.

\section{References}

Abdelaziz, Y. and Hamouine, A. (2008) 'A survey of the extended finite element-review', Computers and Structures, Vol. 86, Nos. 11-12, pp.1141-1151.

Belytschko, T. and Black, T. (1999) 'Elastic crack growth in finite elements with minimal remeshing', International Journal for Numerical Methods in Engineering, Vol. 45, No. 5, pp.601-620.

Bhattacharya, S., Singh, I.V., Mishra, B.K. and Bui, T.Q. (2013) 'Fatigue crack growth simulations of interfacial cracks in bi-layered FGMs using XFEM', Computational Mechanics, Vol. 52, No. 4, pp.799-814.

Chen, L., Rabczuk, T., Bordas, S.P.A., Liu, G.R., Zeng, K.Y. and Kerfriden, P. (2012) 'Extended finite element method with edge-based strain smoothing (ESm-XFEM) for linear elastic crack growth', Computer Methods in Applied Mechanics and Engineering, Vols. 209-212, No. 0, pp.250-265.

Fajdiga, G. and Zafošnik, B. (2015) 'Determining a kink angle of a crack in mixed mode fracture using maximum energy release rate, SED and MTS criteria', Journal of Multidisciplinary Engineering Science and Technology (JMEST), Vol. 2, No. 1, pp.356-362.

Fayed, A.S. (2017) 'Numerical analysis of mixed mode I/II stress intensity factors of edge slant cracked plates', Engineering Solid Mechanics, pp.61-70.

Ismail, A.E., Ghazali, M.Z.M. and Nor, N.H.M. (2017) 'Mode I stress intensity factors of slanted cracks in plates', IOP Conf. Series: Materials Science and Engineering, Vol. 165, No. 1, pp. $1-8$.

Khatri, K. and Lal, A. (2018) 'Stochastic XFEM fracture and crack propagation behavior of an isotropic plate with a hole emanating radial cracks subjected to various in-plane loadings', Mechanics of Advanced Materials and Structures, Vol. 25, No. 9, pp.732-755.

Kirthan L.J., Hegde, R., Girisha, V.A. and Kumar, R.G. (2016) 'Evaluation of mode 1 stress intensity factor for edge crack using displacement extrapolation method', International Journal of Materials and Structural Integrity, Vol. 10, Nos. 3/4, pp.11-22. 
Kumar, S., Singh, I.V., Mishra, B.K. and Rabczuk T. (2015) 'Modeling and simulation of kinked cracks by virtual node XFEM', Computational Methods in Applied Mechanics and Engineering, Vol. 283, No. 0, pp.1425-1466.

Liu, X.Y., Xiao, Q.Z. and Karihaloo, B.L. (2004) 'XFEM for direct evaluation of mixed mode SIFs in homogeneous and bi-materials', International Journal for Numerical Methods in Engineering, Vol. 59, No. 8, pp.1103-1118.

Möes, N., Dolbow, J. and Belytschko, T. (1999) 'A finite element method for crack growth without remeshing', International Journal for Numerical Methods in Engineering, Vol. 46, No. 1, pp.131-15.

Pais, M.J. (2011) Variable Amplitude Fatigue Analysis using Surrogate Models and Exact XFEM Reanalysis, PhD dissertation, University of Florida, USA, pp.1-194.

Pathak, H., Singh, A. and Singh I.V. (2012) 'Numerical simulation of bi-material interfacial cracks using EFGM and XFEM', International Journal of Mechanics and Materials in Design, Vol. 8, No. 1, pp.9-36.

Sukumar, N., Chopp, D.L., Möes, N. and Belytscko T. (2001) 'Modelling holes and inclusions by level sets in the extended finite element method', Computer Methods in Applied Mechanics and Engineering, Vol. 190, Nos. 46-47, pp.6183-6200. 